American Journal of Immunology 4 (2): 23-25, 2008

ISSN 1553-619X

(C) 2008 Science Publications

\title{
An Analysis of a Severe Neck Pain Experienced in a Polymyositis Patient
}

\author{
${ }^{1}$ R.M. Golding and ${ }^{2}$ L.G.F. Giles \\ ${ }^{1}$ The University of New South Wales, Sydney, New South Wales 2052, Australia \\ ${ }^{2}$ Murdoch University,South Street, Murdoch, Western Australia 6150, Australia
}

\begin{abstract}
Following a twisting strain with immediate pain in the neck of a polymyositis patient we detail the analysis of a specific range of data gathered before and after the event. The results reinforce that in this case that the creatine kinase isoenzyme and cardiac Troponin $T$ cannot be used as an indicator of myocardial disease.
\end{abstract}

Key words: Polymyositis, prednisolone, heart rate, erythrocyte sedimentation rate, creatine kinase, creatine kinase isoenzyme, cardiac troponin $\mathrm{T}$.

\section{INTRODUCTION}

Recently we published a research ${ }^{[1]}$ that described an analysis of the creatine kinase isoenzyme and cardiac troponin $\mathrm{T}$ levels in a polymyositis patient. From an extensive model developed to interpret physiological processes we were able to show that the observed elevated creatine kinase-myoglobin (CK-MB) and serum concentration of cardiac troponin $\mathrm{T}(\mathrm{cTnT})$ values for a particular patient are associated with the inflammatory disease, polymyositis and not only myocardial injury. The analysis is focussed particularly on period (i) 27 July 2001 to 3 December 2007, period (ii) 8 August 2001 to 3 December 2007 and period (iii) 28 March 2007 to 3 December 2007 bearing in mind that we have an extensive range of data over a fourteen year period.

On 30 March our patient experienced a twisting strain with immediate pain in the neck due to attempting to shift a heavy object whilst sitting; the patient also suffered breathing problems ie. with only very shallow breathing being possible as well as great difficulty in swallowing. The following morning the pain was so severe that an osteopath was consulted. Following a clinical examination of the neck a diagnosis was made of an acute somatic dysfunction of the right cervico-thoracic joint complex, with severe pain over branches of regional nerves, including the brachial and long thoracic nerves with associated protective muscle spasm. This spasm was considered to be caused either by muscle inflammation and or zyapophysial facet joint possible synovitis.
The response to mobilising treatment with massage of the posterior neck muscles on two occasions by the osteopath was significant and rapid relief followed with a gradual resolution of pain over one to two weeks.

\section{MATERIALS AND METHODS}

After many years of research, we developed a way to determine the appropriate drug regimen to minimize muscle damage in a particular polymyositis patient ${ }^{[1]}$. This regimen was adopted in 2001 that included 62.5 $\mathrm{mg}$ prednisolone every second day, $2 \mathrm{mg}$ of odrik (trandolapril) and three natural products by Mannatech, namely, $450 \mathrm{mg}$ Ambrotose, three Plus tablets and one PhytAloe tablet per day.

Systolic pressure, diastolic pressure and heart rate were measured on a daily basis. Prednisolone was administered in the morning of day one and blood pressure and heart rate were recorded $12 \mathrm{hrs}$ later and then 36 hrs later on day 2.

Erythrocyte sedimentation rate (ESR), serum creatine kinase $(\mathrm{CK})$, creatine kinase isoenzyme myocardium (CK-MB) and cardiac troponin $\mathrm{T}(\mathrm{cTnT})$ were recorded every six weeks by the Queensland Medical Laboratories.

From the 1 Jan 2001 to 31 Dec 2007 the patient's mean systolic pressure, over this period, was $144.74 \pm 10.49 \mathrm{mmHg}$, mean diastolic pressure was $79.25 \pm 6.58 \mathrm{mmHg}$ and the mean pulse pressure was $65.49 \pm 9.21 \mathrm{mmHg}$.

The heart rate results for day 1 and day 2, over the same period, are for day 1 are $92.09 \pm 8.50$ per min and for day $279.03 \pm 7.95$ per min. Although there are variations within the day 1 and day 2 values, the 
average difference, on a yearly basis, is a constant of $13.07 \pm 1.22$ per min. This reinforces that we have no evidence of any changes taking place prior to this study.

By contrast to the previous study ${ }^{[1]}$, we shall be focussing on a limited range of data from the 14 Jan 2008 to the 30 Jun 2008.

\section{RESULTS AND DISCUSSION}

To explore the effect further, we shall examine the ESR, CK, CK-MB and Troponin T levels gathered in 2008 before and after the 30 March 2008 incident. The results are shown in Table 1.

From Table 1 we note the major changes that occurred following the neck problem in the ESR, CK$\mathrm{MB}$ and Troponin T values.

We note the significant increase in the cardiac troponin T levels recorded on 7 April 2008.

Furthermore, by 30 June 2008, the ESR, CK-MB and Troponin $\mathrm{T}$ values had approximately returned to the values prior to the 30 March 2008 incident.

The systolic pressure and the diastolic pressure from 1 Jan 2008 are shown in Fig. 1.

The mean systolic pressure and the mean diastolic pressure are $141.66 \pm 7.42 \mathrm{mmHg}$ and $77.01 \pm 4.82$ $\mathrm{mmHg}$ respectively. The pulse pressure is $64.64 \pm 6.86$ $\mathrm{mmHg}$. This agrees with the results in period (i) in the CK-MB and Trononin $T$ paper ${ }^{[1]}$. In other words, no change was observed for the mean systolic and the mean diastolic pressures.

On the other hand, the difference between the heart rate measured on day 1 and 2 following the prednisolone intake was significantly changed as shown in Fig. 2.

Table 1: The ESR, CK, CK-MB and Troponin T levels gathered in 2008 before and after the 30 March 2008 incident

\begin{tabular}{lllll}
\hline & $\begin{array}{l}\text { ESR } \\
\mathrm{mm} \mathrm{h}^{-1}\end{array}$ & $\begin{array}{l}\mathrm{CK} \\
\mathrm{IU} \mathrm{L}^{-1}\end{array}$ & $\begin{array}{l}\text { CK-MB } \\
\mathrm{IU} \mathrm{L}^{-1}\end{array}$ & $\begin{array}{l}\text { Troponin T } \\
\mathrm{IU} \mathrm{L}^{-1}\end{array}$ \\
\hline 14 Jan 2008 & 19 & 160 & 15.5 & 0.14 \\
25 Feb 2008 & 18 & 120 & 15.0 & 0.12 \\
7 Apr 2008 & 91 & 140 & 7.9 & 1.22 \\
19 May 2008 & 34 & 81 & 12.1 & 0.09 \\
30 Jun 2008 & 20 & 110 & 13.3 & 0.11 \\
\hline
\end{tabular}

The difference between the heart rate measured on day 1 and 2 following the prednisolone intake was reduced to zero following the neck problem but returned to normal within 17 days. Neglecting the results over these seventeen days yields for day $1 \mathrm{a}$ mean of $94.03 \pm 5.57 \mathrm{~min}^{-1}$ and for day 2 a mean of $78.97 \pm 4.87 \mathrm{~min}^{-1}$. These values agree with those given in the CK-MB and Troponin T paper ${ }^{[1]}$.

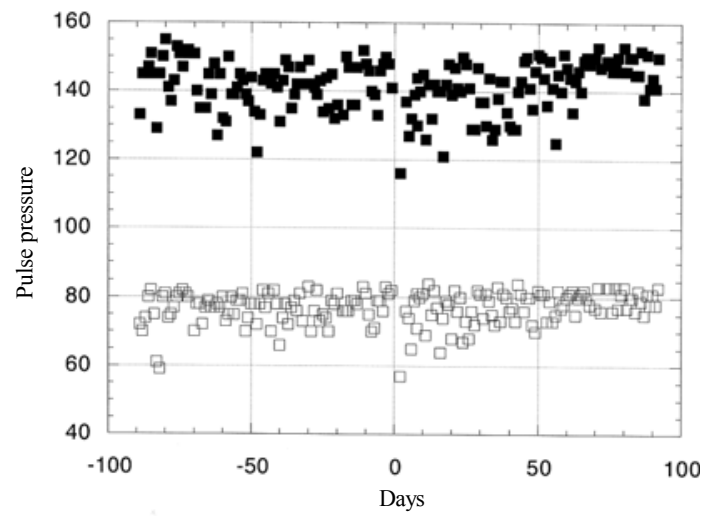

Fig. 1: The systolic pressure and the diastolic pressure in 2008 from 1 January to 30 June in days expressed relative to 30 March 2008. The black squares are the systolic values and the clear squares are the diastolic values.

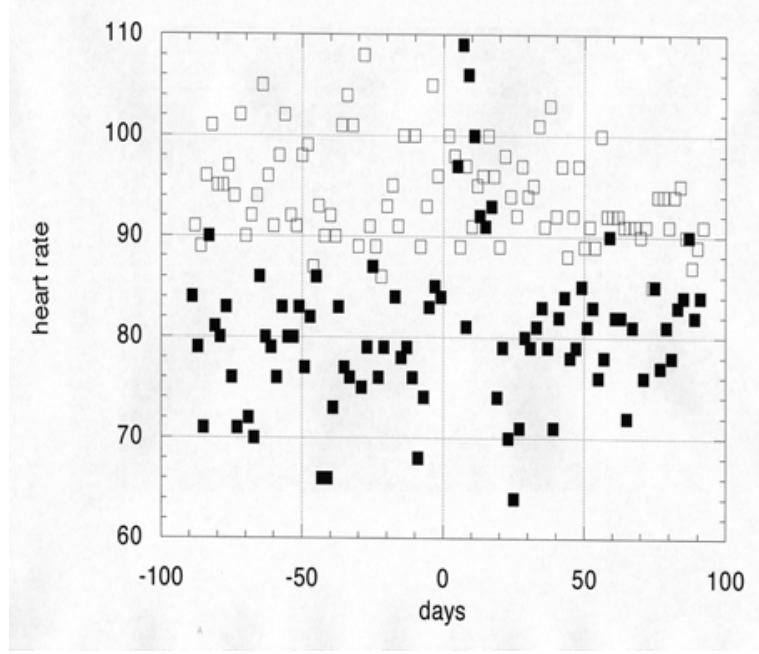

Fig. 2: The heart rate values on day 1 and day 2 following the prednisolone intake in 2008 from 1 January to 30 June in days expressed relative to 30 March 2008. The clear squares are the day 1 heart rate and the black squares are the day 2 heart rate.

The greatly reduced CK-MB value may be due to the significantly decreased chest muscle activity during the period of shallow breathing, as it is known that chest muscles have more CK-MB than other muscles ${ }^{[2]}$, thus the decreased chest muscle activity may have lowered the CK-MB level. 
The daily variation of the systolic and diastolic pressures has not changed as would be expected with a skeletal muscle problem.

\section{CONCLUSION}

In summary, our data, following the twisting strain injury of the neck, appear to reinforce the finding that for patients with skeletal muscle diseases such as polymyositis the plasma levels of the creatine kinase isoenzyme and cardiac troponin $\mathrm{T}$ cannot be used as an indicator of myocardial disease.

\section{ACKNOWLEDGEMENT}

We acknowledge the continued support from the Queensland Medical Laboratories for collecting the required blood samples on a six-weekly basis and for the corresponding pathology reports.

\section{REFERENCES}

1. Golding, R.M., L.G.F. Giles and E.M. Sokoya, 2008. An analysis of the creatine kinase isoenzyme and cardiac Troponin $\mathrm{T}$ levels in a polymyositis patient. Am. J. Immumol., 4: 1-7.

2. American Association for Clinical Chemistry, 2008. CK-MB www.labtestsonline.org/ understanding/analytes/ckmb/test.html. 